\title{
Universal damping mechanism of quantum vibrations in deep sub-barrier fu- sion reactions
}

\author{
Takatoshi Ichikawa ${ }^{1, \star}$ \\ ${ }^{1}$ Center for Nuclear Study, the University of Tokyo, Hongo, Tokyo 113-0033, Japan
}

\begin{abstract}
I demonstrate that the coordinate dependence of the coupling strengths of colliding nuclei is important for describing the deep sub-barrier fusion hindrance. To this end, I firstly show the performance of an extended coupled-channel model by phenomenologically introducing a damping factor in the coupling potential. The damping factor stimulates the damping of quantum vibrations occurring near the touching point of colliding nuclei and introduce a coordinate dependence in the coupling strengths. Next, I directly show the coordinate dependence of the transition strengths of colliding nuclei by microscopically calculating excited states using the random phase approximation method. The obtained transition strengths of colliding nuclei as a function of the center-of-mass distance strongly correlate with the damping factor that reproduces very well the fusion hindrance. This is a direct justification for the concept of the coordinate-dependent coupling strengths. Finally, I conclude that the damping of quantum vibrations near the touching point is the universal mechanism for the deep sub-barrier fusion hindrance.
\end{abstract}

\section{Introduction}

At extremely low incident energies, called the deep subbarrier energies, the steep fall-offs of fusion cross sections compared to the estimations of the standard coupledchannel (CC) model have been observed in a wide range of mass systems (see Ref. [1] for details). These steep falloff phenomena are often referred to as the fusion hindrance. An important observation for understanding this fusion hindrance is that the potential energy at the touching point of the colliding nuclei strongly correlates with the threshold incident energy for the emergence of the fusion hindrance. That is, the fusion hindrance would be associated with dynamics in the overlap region of the two colliding nuclei (see Fig. 1 in Ref. [2]).

To describe these fusion hindrance phenomena, recent theoretical works have been mainly devoted to how to extend the standard CC model in this overlap region. Two different models based on assumptions opposite to each other have been proposed [1]. One is the sudden approach proposed by Mişicu and Esbensen [4, 5]. They constructed a heavy ion-ion potential to stimulate the effect of nuclear incompressibility, which gave a shallow potential pocket. Recently, Simenel et al. used the density-constrained Hartree-Fock method to demonstrate that Pauli repulsion reduces the tunneling probabilities at deep-sub-barrier energies [3].

The other is the adiabatic approach proposed by Ichikawa et al. [6]. In this approach, neck formations between the colliding nuclei are taken into account in the overlap region. Based on this picture, the sudden and adi-

\footnotetext{
^e-mail: ichikawa@cns.s.u-tokyo.ac.jp
}

abatic processes were smoothly jointed by phenomenologically introducing the damping factor in the coupling form factor [7]. That is, a coordinate dependence was introduced in the coupling potential. Later, I showed that the physical origin of the damping factor is the damping of quantum vibrations of the target and the projectile near the touching point using the random-phase approximation (RPA) method for not only the light mass-symmetric ${ }^{16} \mathrm{O}$ $+{ }^{16} \mathrm{O}$ and ${ }^{40} \mathrm{Ca}+{ }^{40} \mathrm{Ca}$ systems [8] but also the massasymmetric ${ }^{16} \mathrm{O}+{ }^{208} \mathrm{~Pb}$ system [9].

In this paper, I demonstrate the importance of the coordinate dependence of the coupling strength using a phenomenological model and examine this assumption by a microscopic RPA calculation. I show that the damping of the quantum vibrations near the touching point occurs, which is a universal mechanism in the deep sub-barrier fusions and is responsible for the fusion hindrance.

In the standard CC model (and the sudden model), the total wave functions are expanded by the basis of isolated nuclei. Then, important input parameters are the excitation energy and the coupling strength for each excitation. The excitation energy is usually taken from the experimental data and the coupling strength is estimated from measured transition probability. However, in the $\mathrm{CC}$ calculations, it is usually assumed that these values are unchanged during the fusion process, even when the two nuclei strongly overlap with each other. This seems to be an unrealistic assumption. As shown in Ref. [8], the single-particle wave functions are drastically changed by level repulsions, which are associated with the neck formations.

So far, many theoretical works for deep sub-barrier fusions have paid attention only how to construct the inter- 
nuclear potential in the overlap region. However, in this paper, I would like to stress that the treatment of the coupling potential in the overlap region is also much important for describing the deep sub-barrier fusion hindrance.

\section{Coordinate-dependent coupling strength phenomenologically introduced by the damping factor}

Before introducing the coordinate dependence in the coupling strength in the standard CC model, I first briefly describe the standard CC model (for details see Refs. [10, 11, 14]).

For heavy-ion fusion reactions, the no-Coriolis approximation is often used $[11,14]$. Then, the CC equations $[11,14]$ are given by

$$
\begin{aligned}
{\left[-\frac{\hbar^{2}}{2 \mu} \frac{d^{2}}{d r^{2}}+\frac{J(J+1) \hbar^{2}}{2 \mu r^{2}}+\right.} & \left.V^{(0)}(r)+\epsilon_{n}-E\right] u_{n}(r) \\
& +\sum_{m} V_{n m}(r) u_{m}(r)=0
\end{aligned}
$$

where $r$ is the radial component of the relative motion coordinate, $\mu$ is the reduced mass, $E$ is the incident energy in the center-of-mass frame, $J$ is the total angular momentum, and $\epsilon_{n}$ is the excitation energy of the $n$-th channel. A bare nuclear potential $V^{(0)}$ consisting of the Coulomb and nuclear interactions is given by $V^{(0)}(r)=$ $Z_{T} Z_{P} e^{2} / r+V_{N}^{(0)}(r)$, where $Z_{T}$ and $Z_{P}$ denote the proton number of the target and projectile, respectively. The matrix elements of the coupling Hamiltonian $V_{n m}$ are calculated with the collective model including the Coulomb and nuclear components.

In coupling matrix elements, I consider only vibrational couplings in this paper. The nuclear coupling Hamiltonian can be generated by changing the target radius in the nuclear potential of $V_{N}^{(0)}$ to a dynamical operator $R_{0} \rightarrow R_{0}+\hat{O}_{\lambda}$. Therefore, the nuclear coupling Hamiltonian is given by $V_{N}\left(r, \hat{O}_{\lambda}\right)=V_{N}^{(0)}\left(r-\hat{O}_{\lambda}\right)[11,14]$. For the vibrational coupling, the operator $\hat{O}_{\lambda}$ is given by $\hat{O}_{\lambda}=\beta_{\lambda} / \sqrt{4 \pi} \cdot R_{T}\left(\alpha_{\lambda 0}^{\dagger}+\alpha_{\lambda 0}\right)$, where $\alpha_{\lambda 0}^{\dagger}$ and $\alpha_{\lambda 0}$ are the creation and annihilation operators of the phonons, respectively, and $R_{T}$ is the radius of the target nucleus. Here, the eigenvalues $\lambda_{\alpha}$ and the eigenvectors $|\alpha\rangle$ of $\hat{O}_{\lambda}$ are given by $\hat{O_{\lambda}}|\alpha\rangle=\lambda_{\alpha}|\alpha\rangle$. The deformation parameter $\beta_{\lambda}$ is an input parameter and can be estimated from an experimental transition probability $B(E \lambda)$ which is given by

$$
\beta_{\lambda}=\frac{4 \pi}{3 Z_{T} R_{T}^{\lambda}} \sqrt{\frac{B(E \lambda) \uparrow}{e^{2}}},
$$

where $e$ is the elementary charge. The matrix elements of the nuclear coupling Hamiltonian are expanded by the eigenvalues and eigenvectors $[10,11,14]$, and thus are defined as

$$
\begin{aligned}
V_{n m}^{(N)} & =\left\langle n\left|V_{N}\left(r, \hat{O}_{\lambda}\right)\right| m\right\rangle-C_{0}(r) \\
& =\sum_{\alpha}\langle n \mid \alpha\rangle\langle\alpha \mid m\rangle V_{N}\left(r, \lambda_{\alpha}\right)-C_{0}(r),
\end{aligned}
$$

where $C_{0}$ is the coupling constant given by $C_{0}(r)=$ $\left\langle 0\left|V_{N}\left(r, \hat{O}_{\lambda}\right)\right| 0\right\rangle$.

A main idea to include the coordinate dependence in the coupling strength is the introduction of the damping factor $\Phi\left(r, \lambda_{\alpha}\right)$ in the coupling form factor. I employ the following form for the nuclear coupling potential with respect to the eigen channel $\alpha$,

$$
V_{N}\left(r, \lambda_{\alpha}\right)=V_{N}^{(0)}(r)+\left[-\frac{d V_{N}^{(0)}}{d r} \lambda_{\alpha}+\frac{1}{2} \frac{d^{2} V_{N}^{(0)}}{d r^{2}} \lambda_{\alpha}^{2}\right] \Phi\left(r, \lambda_{\alpha}\right)
$$

Here,

$$
\Phi\left(r, \lambda_{\alpha}\right)= \begin{cases}1 & r>R_{d}+\lambda_{\alpha} \\ e^{-\left(r-R_{d}-\lambda_{\alpha}\right)^{2} / 2 a_{d}^{2}} & \text { (Two-body region) } \\ & \text { Otherwise } \\ & \text { (Overlap region) }\end{cases}
$$

where $R_{d}$ is the damping dependent touching distance between the target and the projectile defined by $R_{d}=$ $r_{d}\left(A_{T}^{1 / 3}+A_{P}^{1 / 3}\right)$. The coefficients $r_{d}$ and $a_{d}$ are the damping radius and diffuseness parameters. The damping factor $\Phi$ represents the physical process for gradually transitioning from sudden to adiabatic approximations by diminishing the excitation strengths of the target and/or projectile vibrational states after the two colliding nuclei overlap one another. An important modification is that the touching point in the damping factor depends on $\lambda_{\alpha}$, namely, the excitation strength begins to reduce at different distances in each eigen channel.

Although I attempted to apply several functional forms to the damping factor, I found that the form of Eq. (5) can well reproduce various experimental data. Later, I will show the justification of this functional form by a direct calculation of the transition strength using the RPA method.

After these modifications, in the two-body region $(r>$ $R_{d}+\lambda_{\alpha}$ ), the standard CC equations of Eq. (1) work well because $\Phi=1$. Conversely, in the overlapping region $(r<$ $R_{d}+\lambda_{\alpha}$ ), the coupling matrix elements become $V_{n m} \rightarrow$ 0 because $\Phi \rightarrow 0$. Then, the standard CC equations of Eq. (1) are close to the one-dimensional Schrödinger-like equations given by

$$
\begin{array}{r}
{\left[-\frac{\hbar^{2}}{2 \mu} \frac{d^{2}}{d r^{2}}+\frac{J(J+1) \hbar^{2}}{2 \mu r^{2}}+V^{(0)}(r)+\epsilon_{n}-E\right] u_{n}(r)=0} \\
\left(r \ll R_{d}+\lambda_{\alpha}\right) .
\end{array}
$$

If an adiabatic one-body potential $V_{1 \mathrm{bd}}^{(0)}$ is substituted to $V^{(0)}$ in Eq. (6), one can avoid double counting of the CC effects and correctly estimate the tunneling probability in the one-body process. Subsequently, all the physical quantities are smoothly joined.

An example of the application for this model is a calculation for the ${ }^{16} \mathrm{O}+{ }^{208} \mathrm{~Pb}$ system. I incorporate the damping factor in the computer code ccfull [11]. For the ${ }^{16} \mathrm{O}+{ }^{208} \mathrm{~Pb}$ system, I include the coupling to the low-lying $3^{-}$phonon states and the double-octupole phonon excitations for both the ${ }^{16} \mathrm{O}$ and ${ }^{208} \mathrm{~Pb}$ nuclei. In the calculations, 
I adopt the Yukawa-plus-exponential (YPE) potential as a basic heavy ion-ion potential $V^{(0)}(r)$. In order to fit the experimental fusion cross sections, the radius parameter $r_{0}$ is adjusted to be $1.2 \mathrm{fm}$ for ${ }^{16} \mathrm{O}+{ }^{208} \mathrm{~Pb}$ system. For the damping factor, I use $r_{d}=1.255 \mathrm{fm}$ and $a_{d}=1.14 \mathrm{fm}$. The details of all calculations and parameters are given in Ref. [2].

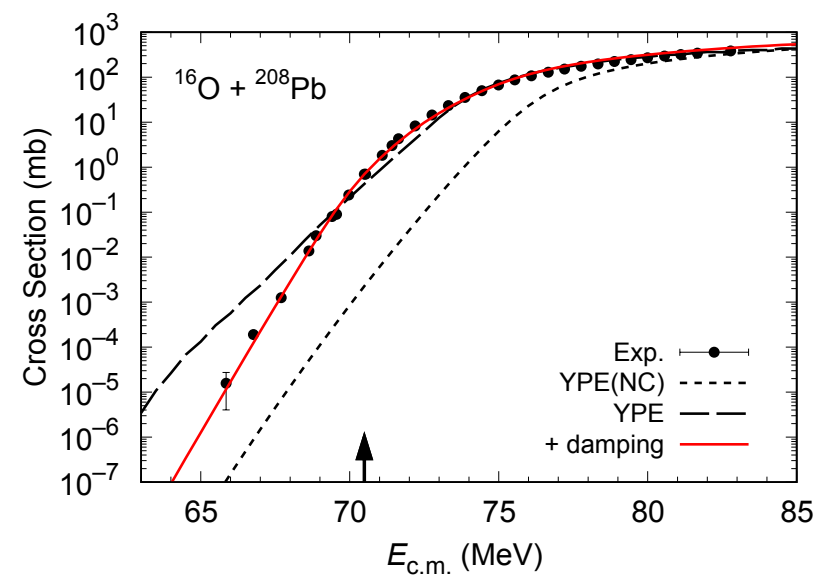

Figure 1. Calculated and experimental fusion cross sections versus incident energies for the ${ }^{16} \mathrm{O}+{ }^{208} \mathrm{~Pb}$ system. The solid circles denote the experimental data $[12,13]$. The solid and long-dashed lines indicate the calculated results with and without the damping factor, respectively. The short-dashed lines indicate the calculated results of no coupling. The arrow indicates the potential energy at the touching point $V_{\text {Touch }}$.

Figure 1 shows the fusion cross sections thus obtained. The fusion cross sections obtained with the damping factor are in good agreement with the experimental data (see the solid line). We see that drastic improvement has been achieved by taking into account the damping of the CC form factors, as compared to the result without the damping factor (the dashed line).

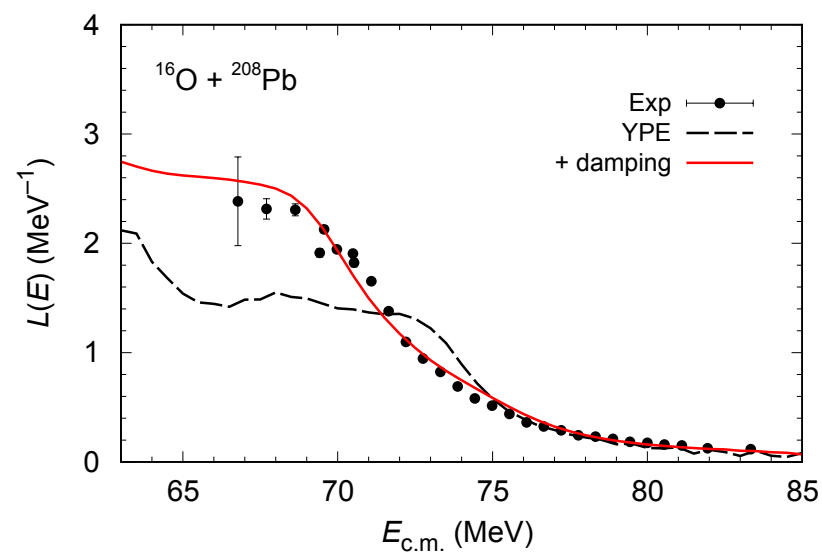

Figure 2. Logarithmic derivative representations of the fusion cross sections versus incident energies for the ${ }^{16} \mathrm{O}+{ }^{208} \mathrm{~Pb}$ system. The solid and dashed lines indicate the results calculated with and without the damping factor, respectively. The solid circles are obtained from the experimental fusion cross sections of Refs. [12, 13].
Figure 2 compares the logarithmic derivatives $d \ln \left(E_{\text {c.m. }} \sigma_{\text {fus }}\right) / d E_{\text {c.m. }}$ of the experimental fusion cross sections with the calculated results. It is again remarkable that only the result with the damping factor achieves nice reproduction of the experimental data. The result with the damping factor becomes saturated below $E_{\mathrm{c} . \mathrm{m} .}=68 \mathrm{MeV}$. The measurement at further lower incident energies for this system will thus provide a strong test for the present adiabatic model.

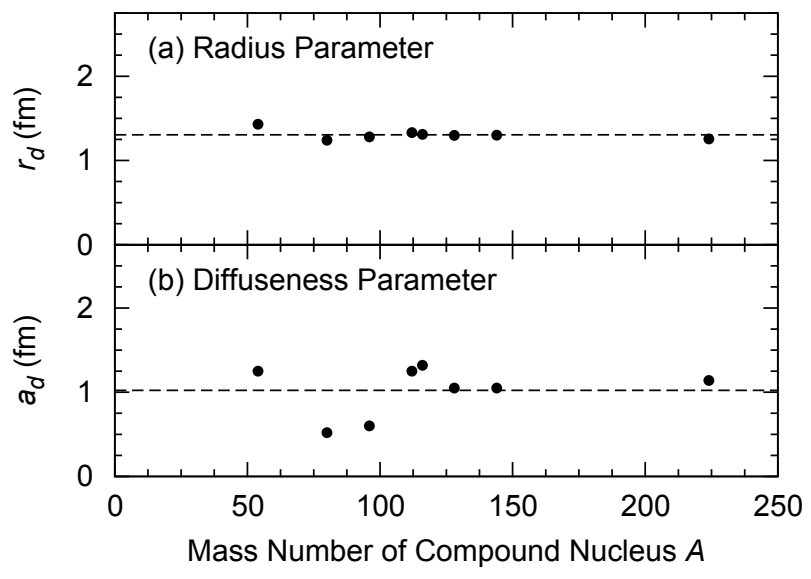

Figure 3. Systematic trends of the (a) radius and (b) diffuseness parameters in the damping factor versus the mass numbers of the compound nuclei for various systems. The solid circles denote the obtained values fitted to the experimental data. The dashed lines indicate the average value of all the obtained values in individual radius and diffuseness parameters.

I have also tested this model in various systems and investigated the systematic trends of the obtained radius and diffuseness parameters in the damping factor. I perform calculations for the medium-heavy mass systems of ${ }^{64} \mathrm{Ni}+{ }^{64} \mathrm{Ni},{ }^{58} \mathrm{Ni}+{ }^{58} \mathrm{Ni}$, and ${ }^{58} \mathrm{Ni}+{ }^{54} \mathrm{Fe}$, the mediumlight mass systems of ${ }^{40} \mathrm{Ca}+{ }^{40} \mathrm{Ca},{ }^{48} \mathrm{Ca}+{ }^{48} \mathrm{Ca}$, and ${ }^{24} \mathrm{Mg}$ $+{ }^{30} \mathrm{Si}$, and the mass-asymmetric systems of ${ }^{48} \mathrm{Ca}+{ }^{96} \mathrm{Zr}$ and ${ }^{16} \mathrm{O}+{ }^{208} \mathrm{~Pb}$. The obtained results are shown in Fig. 3. Clearly, the values of $r_{d}$ are almost constant at around an average value of $1.31 \mathrm{fm}$. Except for a few points, the values of $a_{d}$ are also distributed around an average value of $1.02 \mathrm{fm}$.

Thus, the damping factor strongly correlates with the damping of the transition strength of individual colliding nuclei when they approach one another. In this respect, the damping of the transition strengths would start at the overlapping between the tails of the density distributions for colliding nuclei. That is, the radius parameter of the damping factor would correlate with a range of interactions between the colliding nuclei. This would result in an almost constant value of $r_{d}$ in all the systems.

\section{Direct calculation of coordinate-dependent transition strength using the RPA method}

As shown in the previous section, the damping factor phenomenologically introduced works very well for describ- 
ing the deep sub-barrier fusion hindrance in various systems. The damping factor tells us the importance of the coordinate dependence of the coupling strength. To justify this assumption, I directly calculate the transition strength for ${ }^{16} \mathrm{O}+{ }^{208} \mathrm{~Pb}$ as a function of the center-of-mass distance $R$ using the RPA method.

I calculate the mean-field potential for the ${ }^{16} \mathrm{O}+{ }^{208} \mathrm{~Pb}$ system using the folding procedure with the single Yukawa function [15]. Before the touching point, I assume the spherical shape for both nuclei. After the touching point, I describe the nuclear shapes with the reflection-asymmetric lemniscatoids parametrization [16]. (The parametrization dependence is negligible, because in this paper I do not discuss the strongly overlapping region.) Based on these densities, I also calculate the Coulomb potential. I use the radius for the proton and neutron potentials, $R_{0}$, with $R_{0}=1.27 A^{1 / 3} \mathrm{fm}$, where $A$ is the total nucleon number. The depths of the neutron and proton potentials for individual ${ }^{16} \mathrm{O}$ and ${ }^{208} \mathrm{~Pb}$ nuclei, $V_{T}$ and $V_{P}$, are taken from Ref. [17].

Using the obtained mean-field potentials, I solve the axially-symmetric Schrödinger equation with the spinorbit force and obtain the single-particle wave functions $\phi_{i}(R)$ and the eigen energies $\epsilon_{i}(R)$. The details of the model and the parameters are given in Ref. [9]. Then, the $z$ component of the total angular momentum, $\Omega$, is the good quantum number. Note that the parity is not a good quantum number because the mean-field potential for the whole system breaks the space-reflection symmetry. I expand the single-particle wave functions in terms of the deformed harmonic-oscillator bases in the cylindrical coordinate representation. The deformation parameter of the basis functions is determined so as to cover the target and the projectile. The basis functions with energies lower than $26 \hbar \omega$ are taken into account.

The obtained single-particle energies as a function of the center-of-mass distance $R$ is shown in Fig. 1 of Ref. [9]. As shown in the figure, the single-particle wave functions are drastically changed by level repulsions, which are associated with the neck formations. We can also see extremely strong Coulomb effect of ${ }^{208} \mathrm{~Pb}$ on ${ }^{16} \mathrm{O}$. The singleparticle energies of the $p_{1 / 2}$ and $p_{3 / 2}$ states in ${ }^{16} \mathrm{O}$ remarkably increase with decreasing $R$ due to the increasing Coulomb effect from ${ }^{208} \mathrm{~Pb}$. Then, many level crossings and repulsion between the energy levels of ${ }^{16} \mathrm{O}$ and ${ }^{208} \mathrm{~Pb}$ occur. Below $R=13 \mathrm{fm}$, the Coulomb barrier becomes lower due to the attractive nuclear mean-field potential. Then, the strong mixture of the single-particle states between ${ }^{16} \mathrm{O}$ and ${ }^{208} \mathrm{~Pb}$ starts in many levels, which causes many level splittings seen in the Nilsson diagram.

I now solve the RPA equation at each $R$ for the massasymmetric ${ }^{16} \mathrm{O}+{ }^{208} \mathrm{~Pb}$ system. The RPA equation then reads

$$
\left(\begin{array}{cc}
A & B \\
B^{*} & A^{*}
\end{array}\right)\left(\begin{array}{l}
X^{v}(R) \\
Y^{v}(R)
\end{array}\right)=\hbar \Omega_{v}(R)\left(\begin{array}{cc}
1 & 0 \\
0 & -1
\end{array}\right)\left(\begin{array}{l}
X^{v}(R) \\
Y^{v}(R)
\end{array}\right),
$$

where

$$
\begin{aligned}
& A_{\operatorname{minj}}=\left\langle\phi(R)\left|\left[a_{i}^{\dagger} a_{m}\left[H, a_{n}^{\dagger} a_{j}\right]\right]\right| \phi(R)\right\rangle \\
& B_{\text {minj }}=\left\langle\phi(R)\left|\left[a_{i}^{\dagger} a_{m}\left[H, a_{j}^{\dagger} a_{n}\right]\right]\right| \phi(R)\right\rangle .
\end{aligned}
$$

The symbols $X$ and $Y$ are the forward and backward amplitudes, respectively, $\hbar \Omega$ is the excitation energy, $a^{\dagger}$ (a) indicates creation (annihilation) operator, and $H$ is the HatreeFock Hamiltonian. I calculate the first excited $3^{-}$(octupole vibrational) states of ${ }^{16} \mathrm{O}$ and ${ }^{208} \mathrm{~Pb}$, which give the main contributions in the standard CC calculations. I can easily apply the RPA method to the di-nuclear system, because its wave function is described with a one-center Slater determinant. I take the single-particle levels for each neutron and proton from first to 200th and the coherent superposition of all one-particle one-hole states with excitation energies below $30 \mathrm{MeV}$. I follow the diabatic single-particle configuration corresponding to the ground state of ${ }^{16} \mathrm{O}$. I use the density-dependent residual interaction taken from Ref. [18] and tune it so that the energy of the spurious center-of-mass motion becomes zero. I calculate $B(\mathrm{E} 3)$ values for the RPA solutions with $\Omega=0$ in individual nuclei using the shifted octupole operator, $\widehat{Q}_{30}\left(R-R_{0}^{\prime}\right)$, where $R_{0}^{\prime}$ is the center-of-mass position of the projectile or target nucleus.

At $R=20 \mathrm{fm}$, the obtained first excited octupole modes for ${ }^{16} \mathrm{O}$ and ${ }^{208} \mathrm{~Pb}$ are isolated. When the two nuclei approach each other, however, these modes start to fragment into several states. To evaluate the octupole collective strengths carried by low-energy excitations, I then calculate the energy-weighted sum of $B(E 3)$ strengths. By checking the spectrum of all obtained RPA modes as a function of $R$, I determined to take the sum for octupole excitations with $E \leq 4 \mathrm{MeV}$ and $E \leq 6 \mathrm{MeV}$ for ${ }^{208} \mathrm{~Pb}$ and ${ }^{16} \mathrm{O}$, respectively.

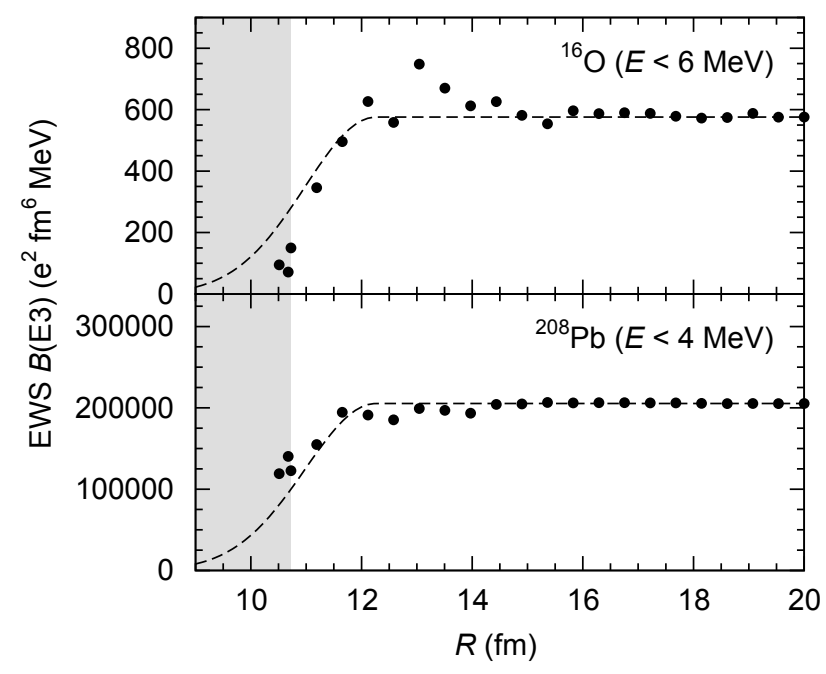

Figure 4. Energy-weighted sums of $B(\mathrm{E} 3)$ for (a) ${ }^{16} \mathrm{O}$ and (b) ${ }^{208} \mathrm{~Pb}$ as functions of $R$. The solid circles show the results of the RPA calculations. The dashed curves represent the damping factor which well reproduces the experimental data of the fusion cross section for ${ }^{16} \mathrm{O}+{ }^{208} \mathrm{~Pb}$. The gray area indicates the overlap region of the colliding nuclei.

Figure 4 shows the $B(\mathrm{E} 3)$ strengths for (a) ${ }^{16} \mathrm{O}$ and (b) ${ }^{208} \mathrm{~Pb}$ as functions of $R$. The calculated values (the solid circles) drastically decrease near the touching point (the boundary between the white and gray areas) in both nuclei. The transition densities and currents for the RPA 
modes with the maximum $B(\mathrm{E} 3)$ at the touching point are depicted in Figs. 2 (b) and (d) of Ref. [9]. These figures indicate that the octupole collectivities of both ${ }^{16} \mathrm{O}$ and ${ }^{208} \mathrm{~Pb}$ are considerably diminished by each colliding partner.

To see the correlation with the damping factor phenomenologically introduced in the CC calculation, I compare the calculated results with the damping factor that well reproduced the experimental data of the fusion cross section for ${ }^{16} \mathrm{O}+{ }^{208} \mathrm{~Pb}$ shown in the previous section. In this case, the largest eigenvalue of $\lambda_{\alpha}$ is $1.46 \mathrm{fm}$. In Figs. 4 (a) and (b), the dashed curves represent the damping factor with these parameters normalized at $R=20 \mathrm{fm}$. We can see that the damping factor strongly correlates with the calculated energy-weighted sums of $B(\mathrm{E} 3)$ in the lowenergy region, which clearly indicates that the damping of the quantum vibrations indeed occurs when the colliding nuclei approach each other. The assumed functional form of Eq. (5) is also justified. These results clearly show the importance of the coordinate dependence of the coupling strength.

This assumption has been also examined in various systems. In Ref. [8], the damping of quantum vibrations was obtained in the ${ }^{16} \mathrm{O}+{ }^{16} \mathrm{O}$ and ${ }^{40} \mathrm{Ca}+{ }^{40} \mathrm{Ca}$ systems. This causes by the drastic change of single-particle wave functions constituting the low-energy collective excitations. Therefore, the damping of quantum vibrations would be the universal mechanism in all deep sub-barrier fusion reactions.

\section{Summary}

In summary, I have demonstrated the damping of the quantum octupole vibrations of both ${ }^{16} \mathrm{O}$ and ${ }^{208} \mathrm{~Pb}$, when they approach each other. That is, the coordinate dependence of the coupling strength plays an important role in describing the deep sub-barrier fusion hindrance. I have examined the coordinate-dependent coupling strength by introducing the damping factor in the coupling potential in the standard $\mathrm{CC}$ model. The damping factor suppresses the transition strength near the touching point of colliding nuclei. The $\mathrm{CC}$ calculations including the damping factor reproduce well the fusion hindrance in various systems. The obtained parameter $r_{d}$, which is the radius parameter in the damping factor, is almost constant in all systems.

I have also examined the coordinate dependence of the transition strength by calculating the excited states of ${ }^{16} \mathrm{O}$ and ${ }^{208} \mathrm{~Pb}$ as a function of $R$ using the RPA method. The obtained $B(\mathrm{E} 3)$ strengths are substantially damped near the touching point of the colliding nuclei. The obtained energy-weighted sum of $B(\mathrm{E} 3)$ in the low-energy region exhibits a strong correlation with the damping factor that reproduces well the experimental data of the fusion cross section for ${ }^{16} \mathrm{O}+{ }^{208} \mathrm{~Pb}$. This is a clear evidence that the damping of the quantum octupole vibrations indeed occur near the touching point in the deep sub-barrier fusion reactions.

The drastic change of single-particle wave functions constituting the low-energy collective excitations would commonly occur in all deep sub-barrier reactions. Therefore, the damping of quantum vibrations, that is, the coordinate-dependent coupling strengths, in both the target and projectile near the touching point seems to be the universal mechanism causing the fusion hindrance, which should be taken into account in the standard CC model.

I thank K. Matsuyanagi, K. Hagino, and A. Iwamoto for collaborations and useful discussions.

\section{References}

[1] B.B. Back et al., Rev. Mod. Phys. 86, 317 (2014).

[2] T. Ichikawa, Phys. Rev. C 92, 064604 (2015).

[3] C. Simenel, A. S. Umar, K. Godbey, M. Dasgupta, and D. J. Hinde Phys. Rev. C 95, 031601(R)

[4] Ş Mişicu and H. Esbensen, Phys. Rev. Lett. 96, 112701 (2006); Phys. Rev. C 75, 034606 (2007).

[5] H. Esbensen and Ş Mişicu, Phys. Rev. C 76, 054609 (2007).

[6] T. Ichikawa, K. Hagino, and A. Iwamoto, Phys. Rev. C 75, 057603 (2007).

[7] T. Ichikawa, K. Hagino, A. Iwamoto, Phys. Rev. Lett. 103, 202701 (2009); EPJ Web Conf. 17, 07001 (2011).

[8] T. Ichikawa, K. Matsuyanagi, Phys. Rev. C 88, 011602(R) (2013).

[9] T. Ichikawa, K. Matsuyanagi, Phys. Rev. C 92, 021602(R) (2015).

[10] H. Esbensen and S. Landowne, Phys. Rev. C 35, 2090 (1987).

[11] K. Hagino, N. Rowley, and A.T. Kruppa, Comp. Phys. Comm. 123, 143 (1999).

[12] M. Dasgupta et al., Phys. Rev. Lett. 99, 192701 (2007).

[13] C. R. Morton, A. C. Berriman, M. Dasgupta, D. J. Hinde, and J. O. Newton, K. Hagino, and I. J. Thompson, Phys. Rev. C60, 044608 (1999).

[14] K. Hagino and N. Takigawa, Prog. Theor. Phys. 128, 1061 (2012).

[15] M. Bolsterli et al., Phys. Rev. C 51050 (1972).

[16] G. Royer and B. Remaud, Nucl. Phys. A444, 477 (1985).

[17] P. Möller et al., At. Data Nucl. Data Tables 59 (1995) 185.

[18] S. Shlomo and G. Bertsch, Nucl. Phys. A 243, 507 (1975). 